\title{
LINES OF FULL RANK MATRICES IN LARGE SUBSPACES
}

\author{
Clément de Seguins PaZzis
}

Abstract. Let $n$ and $p$ be non-negative integers with $n \geqslant p$, and $S$ be a linear subspace of the space of all $n$ by $p$ matrices with entries in a field $\mathbb{K}$. A classical theorem of Flanders states that $S$ contains a matrix with rank $p$ whenever $\operatorname{codim} S<n$.

In this article, we prove the following related result: if $\operatorname{codim} S<n-1$, then, for any non-zero $n$ by $p$ matrix $N$ with rank less than $p$, there exists a line that is directed by $N$, has a common point with $S$ and contains only rank $p$ matrices.

Mathematics subject classification (2010): 15A03, 15A30.

Keywords and phrases: Full rank, matrices, dimension, Flanders's theorem.

\section{REFERENCES}

[1] J. Dieudonné, Sur une généralisation du groupe orthogonal à quatre variables, Arch. Math. vol. 1 (1948), 282-287.

[2] H. Flanders, On spaces of linear transformations with bounded rank, J. Lond. Math. Soc., vol. 37 (1962), 10-16.

[3] R. Meshulam, On the maximal rank in a subspace of matrices, Quart. J. Math. Oxford (2), vol. 36 (1985), 225-229.

[4] C. DE Seguins PAZZIS, Range-compatible homomorphisms on matrix spaces, Linear Algebra Appl., vol. 484 (2015), 237-289.

[5] C. DE SEguins PAZZIS, The affine preservers of non-singular matrices, Arch. Math., vol. 95 (2010), $333-342$.

[6] C. De Seguins Pazzis, The Flanders theorem over division rings, Linear Algebra Appl., vol. 493 (2016), 313-322. 